\title{
Scaling Behavior and Equilibrium Lengths of Knotted Polymers
}

\author{
Eric Rawdon, ${ }^{\dagger}$ Akos Dobay, ${ }^{*}$ John C. Kern, ${ }^{\S}$ Kenneth C. Millett, ${ }^{*, l}$ Michael Piatek, ${ }^{\perp}$ \\ Patrick Plunkett, ${ }^{\#}$ and Andrzej Stasiak ${ }^{\nabla}$
}

\author{
Department of Mathematics, University of St. Thomas, St. Paul, Minnesota 55105, \\ Ludwig-Maximilians-Universitat, Biozentrum, Grosshadernerstrasse 2, 82152 Munich, Germany, Department \\ of Mathematics and Computer Science, Duquesne University, Pittsburgh, Pennsylvania 15282, Department \\ of Mathematics, University of California, Santa Barbara, Santa Barbara, California 93106, Department of \\ Computer Science and Engineering, University of Washington, Seattle, Washington 98195, Department of \\ Mathematics, University of California, Santa Barbara, Santa Barbara, California 93106, and Faculty \\ of Biology and Medicine, Center for Integrative Genomics, University of Lausanne, Lausanne \\ CH 1015, Switzerland
}

Received January 11, 2008; Revised Manuscript Received March 14, 2008

\begin{abstract}
We use numerical simulations to investigate how the chain length and topology of freely fluctuating knotted polymer rings affect their various spatial characteristics such as the radius of the smallest sphere enclosing momentary configurations of simulated polymer chains. We describe how the average value of a characteristic changes with the chain size and how this change depends on the topology of the modeled polymers. Although the scaling profiles of a spatial characteristic for distinct knot types do not intersect (at least, in the range of our data), the profiles for nontrivial knots intersect the corresponding profile obtained for phantom polymers, i.e., those that are free to explore all available topological states. For each knot type, this point of intersection defines its equilibrium length with respect to the spatial characteristic. At this chain length, a polymer forming a given knot type will not tend to increase or decrease, on average, the value of the spatial characteristic when the polymer is released from its topological constraint. We show interrelations between equilibrium lengths defined with respect to spatial characteristics of different character and observe that they are related to the lengths of ideal geometric configurations of the corresponding knot types.
\end{abstract}

\section{Introduction}

Depending on the type of interactions between independent segments of a polymeric chain, one observes three fundamental types of polymer behavior: self-attractive, self-repulsive, and neutral. ${ }^{1}$ Self-attractive behavior is observed when a diluted polymer is suspended in a so-called poor solvent. Under these conditions, individual chains of a polymer collapse into compact ellipsoidal blobs whose overall dimension, such as the radius of gyration, grow with the cube root of the chain length and, therefore, show a scaling exponent $v=1 / 3$. Self-repelling behavior occurs when diluted polymers are suspended in a good solvent. Under these conditions, segments of a fluctuating chain that come closer to each other than the Debye screening length repel each other. The interplay between the repulsive forces and entropic effects causes polymer chains to adopt loose coil configurations. The increase of overall dimension of the coils in terms of their chain length is characterized by the scaling exponent with the widely accepted value $\approx 0.588,{ }^{2}$ although simulations from ref 3 indicate a more precise value of $v=$ $0.5874 \pm 0.0002$. The neutral behavior is observed for diluted linear polymers in so-called $\Theta$-solvents where segments that are not connected to each other neither attract nor repel each other. Under these conditions, entropic effects shape the polymer

\footnotetext{
* Corresponding author.

${ }^{\dagger}$ Department of Mathematics, University of St. Thomas. E-mail: ejrawdon@stthomas.edu.

Ludwig-Maximilians-Universitat. E-mail: akos.dobay@ physik.lmu.de.

${ }^{\S}$ Department of Mathematics and Computer Science, Duquesne University.E-mail: kern@mathcs.duq.edu.

"Department of Mathematics, University of California, Santa Barbara, Santa Barbara, California 93106. E-mail: millett@math.ucsb.edu.

${ }^{\perp}$ Department of Computer Science and Engineering, University of Washington. E-mail: piatek@cs.washington.edu.

\# Department of Mathematics, University of California, Santa Barbara. E-mail: plunkett@umail.ucsb.edu.

${ }^{\nabla}$ Faculty of Biology and Medicine, Center for Integrative Genomics, University of Lausanne. E-mail: Andrzej.Stasiak@unil.ch.
}

and the loose coils increase their overall dimensions with the square root of the chain length (scaling exponent $v=0.5$ ). The same scaling behavior with the exponent $v=0.5$ is also observed in highly concentrated polymers suspended in a good solvent. Under these conditions, the melt phase forms where the repulsive interactions acting on any segment are balanced so that the shape of each polymer chain is determined only by entropic contributions. ${ }^{1}$

Numerical simulations of polymers using a simple model of random walks composed of freely jointed segments of equal length with no thickness exhibit many essential features of the spatial behavior of modeled macromolecules under different scaling regimes. Numerical modeling reveals that when segments of random chains have no thickness and are permitted to pass through each other during simulated fluctuations, the modeled macromolecules behave like neutral polymers and show the scaling exponent $v=0.5$. Interestingly, when the simulated circular macromolecules (which have no thickness) are not permitted to pass through each other and therefore reflect the behavior of circular macromolecules that conserve their topology, the scaling behavior changes to that of self-avoiding polymers with the scaling exponent $v \approx 0.588$. This topological influence on the statistical behavior of polymers is the subject of theoretical and numerical studies that addressed, among others, how the growth rate of the radius of gyration, the average crossing number, the total curvature and the total torsion are affected by the topological state of modeled macromolecules. ${ }^{2,4-9}$ In this paper, we look at the effect of topology on additional spatial characteristics of modeled polymers and determine which of these provide statistically distinct spatial information.

The subject of topology in polymer structure has a significant research history beginning with the conjecture of Frisch and Wasserman ${ }^{10}$ and Delbrück ${ }^{11}$ that the probability of knotting approaches unity with increasing number of edges in polygons 


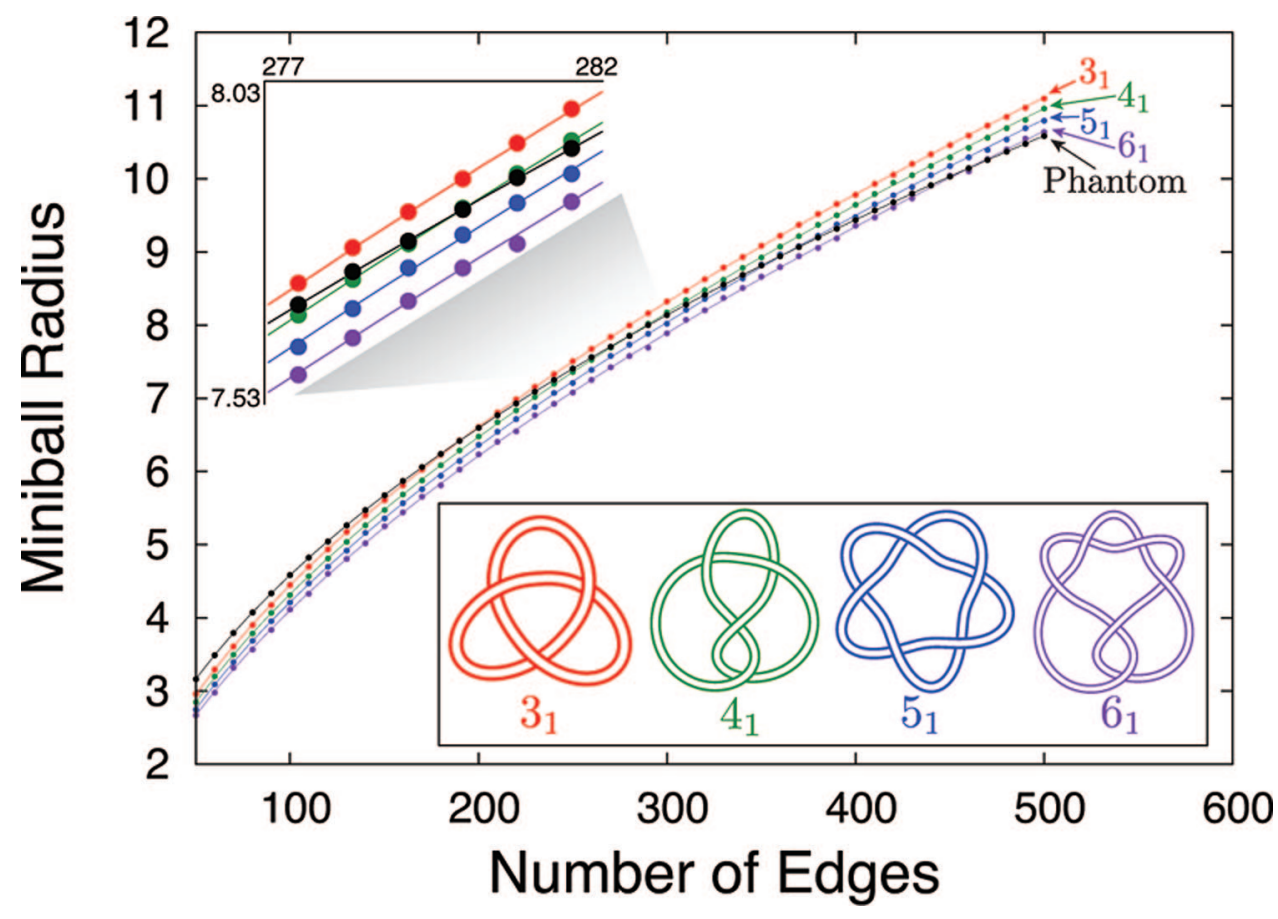

Figure 1. The scaling profile of the average miniball radius (MBR) for all generated random polygons taken together (phantom polygons, black curve) intersects the respective profiles of random configurations of various knot types. The intersection points define the equilibrium length for a given knot type with respect to a given characteristic (miniball radius in this case). The blown up region shows the intersection between the profile for $4_{1}$ knots (green curve) with that of phantom polygons (black curve). Standard diagrams of some of the analyzed knot types are shown together with their Alexander-Briggs notation. In the notation, the first number indicates the minimal number of crossings a given knot can have in a standard projection and the second number indicates its tabular position among the knots with the same number of crossings in standard tables of knots. ${ }^{32,33}$ Notice that for chiral knots such as $3_{1}, 5_{1}$, and $6_{1}$, we group together the left- and right-handed versions of these knots in our statistical analysis.

or chain length in ring polymers. This conjecture was proved, in the lattice case, by Sumners and Whittington ${ }^{12}$ and by Pippenger, ${ }^{13}$ and, in 3 -space models, by Diao ${ }^{14}$ and Diao, Pippenger, and Sumners. ${ }^{15}$ Following this, there have been numerous explorations of the specific parameters describing the finite range and asymptotic range probability distribution functions for knotting and the occurrence of the individual knot type. $^{16-29}$

Of particular interest in our present study is the equilibrium length ${ }^{5-8,30,31}$ associated to the individual spatial structural measures. The equilibrium length of different knot types of polymers, under $\Theta$-conditions or in the melt phase, can be determined through numerical simulations of random polygon models. The equilibrium length associated to a given spatial characteristic of a knot type is defined to be the intersection of the scaling profile of the characteristic measure of the knot type with the scaling profile for the same measure over the entire collection of polygons, i.e., for phantom polygons. For example, in measuring the radius of gyration, the scaling function for very long polymers has been determined to be $A n^{v}$, where $n$ is the number of edges, the amplitude $A$ is dependent upon the model, and the scaling exponent $v$ is independent of the model. For phantom polygons, $v$ is 0.5 , and for self-avoiding polygons, such as those of a specific knot type, $v$ is 0.588 .

The physical meaning of the equilibrium length can be conveniently explained using Figure 1 . Assume that we have a knotted DNA molecule forming a figure-eight knot $\left(4_{1}\right)$ and that this molecule has a chain size corresponding to 100 statistical segments. The average radius of the smallest enclosing ball, the so-called miniball, ${ }^{34}$ for $4_{1}$ knots with 100 edges is approximately $4.3 l$ (see Figure 1), where $l$ is the length of a statistical segment. What happens to this DNA molecule in the presence of DNA topoisomerases ${ }^{35,36}$ that remove the topological constraint and act in a globally random manner? The molecule expands to occupy, on average, a miniball of radius approximately $4.6 l$, i.e., the average miniball radius for 100 statistical segment-long cyclic molecules with unrestricted topology (whose profile is traced by the black line in Figure 1). Now consider a figure-eight DNA molecule with 500 statistical segments. This molecule occupies, on average, a miniball of radius $11.0 l$. When released from the topological constraint, the molecule decreases its overall size and fits, on average, into a miniball of radius $10.6 l$. What happens, then, to a figure-eight DNA molecule with 280 statistical segments upon removing the topological constraint? The average miniball radius of this molecule does not change as a consequence of the process since 280 segments is the equilibrium length with respect to the miniball radius for polymer molecules forming figure-eight knots. As is shown in Figure 1, the equilibrium lengths with respect to the miniball radius have different values for different knot types, where the more complex knots have higher equilibrium lengths. Below their respective miniball equilibrium lengths, the knotted molecules with a given knot type have a tendency to expand upon release from a topological constraint while the opposite is true for knotted polymer molecules whose chain length exceeds the equilibrium length.

Notice that in the example discussed above, we have considered topoisomerases that do not use the energy of ATP hydrolysis to promote strand passages and are, therefore, unable to shift the thermodynamic equilibrium. For example, topoisomerase I of Escherichia coli, acting on double-stranded circular DNA with single-stranded cuts, brings this DNA to topological equilibrium at the given solvent conditions. ${ }^{37}$ Better known type-II DNA topoisomerases that use the energy of ATP hydrolysis are able to change the topological equilibrium and reduce the steady-state knotting level by more than 1 order of magnitude below the knotting level of the thermodynamic equilibrium. ${ }^{38-40}$ 
Grosberg et al. 8,30,31 introduced the overknotted and underknotted regimes to refer to a polymer with a given knot type. In the overknotted regime (relatively short chain size for this knot type), a polymer with a given knot type would have a tendency to simplify its knot type upon release from the topological constraint. In the underknotted regime (relatively long chain size for this knot type), the polymer would have the tendency to increase the knot complexity.

In refs 5 and 6 , the equilibrium length was computed for two quantities, the average crossing number and the radius of gyration. In those papers, the equilibrium values with respect to these two quantities were close enough that one might suspect that the equilibrium length is a universal quantity for a knot type within a given model, independent of the choice of spatial characteristic quantity. In ref 7, the equilibrium lengths were computed for the total curvature and the total torsion and compared to the lengths at which different knot types attained their maximum probability. While the equilibrium lengths defined with respect to the total curvature and total torsion are very similar to each other for each knot type, they are significantly different from the equilibrium lengths measured with respect to either the radius of gyration or the average crossing number. ${ }^{5,6}$ A goal of this research is to determine the extent to which these previously characterized families of equilibrium lengths are comparable to other spatial characteristics and whether there are additional distinct families of spatial characteristics. Distinct families would appear to capture distinct facets of the spatial structure of the polygons thereby providing new perspectives on the structure of the polymers they model.

\section{Quantities Studied}

The radius of gyration, ${ }^{5}$ average crossing number, ${ }^{6}$ total curvature, and total torsion ${ }^{7}$ are four quantities of spatial polygons whose behavior has been observed to be correlated to experimental observations of polymers and other macromolecules such as DNA. Are there are other spatial attributes of random polygons which capture independent information and which also correlate with experimental observations? In the research reported here, we have selected a wide range of such attributes for study. We report on our investigation of the equilibrium lengths and scaling properties of random equilateral polygons with the respect to the following spatial characteristics:

The average crossing number (ACN) is the average of the number of crossings seen when the polygon is projected in every direction. For polygons, the average crossing number is computed in closed form as proposed by Banchoff ${ }^{41}$ and implemented by Sullivan in Brakke's Surface Evolver ${ }^{42}$ (see also ref 43).

The radius of gyration ( $\mathrm{RGN}$ ) is a standard measure of the overall dimensions of polymer chains and corresponds to the root-mean-square distance of the vertices of the polygon from their common center of mass.

The miniball is the smallest sphere that contains all vertices of the polygon and is characterized by the miniball radius (MBR) computed using ref 34.

The thickness radius (THI) is a measure of the extent to which the polymer is self-avoiding (for details, see refs 44-46). Roughly speaking, it is the radius of a thickest non selfintersecting tube about the polymer. Knot configurations with maximal thickness radius for a fixed length are called ideal or tight knots and have been shown to predict some of the physical and statistical properties of knotted polymers (see e.g., refs 47-51).

The dimensions of a smallest standard box enclosing the vertices of a polygon were used as a measure of size in ref 52 where the box length (BXL) is the maximal distance between any pair of vertices. The box width (BXW) is the maximum distance between any pair of vertices in a projection on a plane perpendicular to the chord defining the box length. The box height $(\mathrm{BXH})$ is then the length of the one-dimensional projection of the analyzed polygon on the line perpendicular to the line segment defining the box width. In addition to linear dimensions, we also measure the box surface area (BXA) and box volume (BXV).

The skinny box is a more "economical" box where the longest orthogonal distances are not placed along the major axes of the orthogonal box. The skinny box height, for example, is the shortest distance between parallel walls which contain the polygon. As above, we measure the skinny box length, width, height, surface area, and volume, denoted SBL, SBW, SBH, SBA, and SBV respectively.

The convex hull is the spatial polyhedron that one would obtain by shrink-wrapping the set of points corresponding to the vertices of the polygon. We measure the surface area (CHA) and volume (CHV) of the convex hull using qhull. ${ }^{53}$

The equilibrium lengths for the following two quantities were computed in ref 7 and are in the tables below. We include them for completeness.

The total curvature (TCU) is the sum of the turning angles at the vertices and is a discrete analog of the total curvature of a curve in geometry. ${ }^{54,55}$

The total torsion (TTO) is the sum of the angles between the two planes incident each edge. The first plane is determined by the edge and the previous edge and the second plane is determined by the edge and the following edge. This is a discrete analog of the total torsion of a curve in geometry. Figure 2 shows the miniball, convex hull, box, and skinny box of a figureeight knot.

A detailed description of these spatial characteristics is provided in the Supporting Information.

\section{Computations}

We analyze equilateral random polygons from 50 edges to 500 edges by increments of 10 . For each number of edges, we generated 400000 polygons using the hedgehog algorithm ${ }^{56}$ and classified these polygons by their HOMFLY polynomials ${ }^{57}$ using the program of Ewing and Millett. ${ }^{58} \mathrm{We}$ analyze the spatial information for the $3_{1}, 4_{1}, 5_{1}, 5_{2}, 6_{1}, 6_{2}$, and $6_{3}$ knots as well as phantom polygons (i.e., the entire knot population) at each number of edges. Because the average spatial values for rightand left-handed versions of a chiral knot should be the same, we group them together to provide a statistically robust data set. A more detailed explanation of the polygon generation can be found in ref 7 .

We next determine, for each spatial quantity, the number of edges for which the graph of an individual knot type intersects the graph for the entire knot population, the phantom polygons. To do so, we fit the data with functions that capture the behavior of the graphs. With the exception of the average crossing number and thickness radius, a function of the form

$$
n^{d v}(A+B / \sqrt{n}+C / n)
$$

is used. This scaling was proposed by Orlandini et al. ${ }^{18}$ The value of power $d v$ depends on the "dimension" of the quantity (length-scale quantities have $d=1$, surface area quantities have $d=2$, and volume quantities have $d=3$ ) and whether we are fitting phantom polygons $(v=0.5)$ or a fixed knot type $(v=$ 0.588 ). We use gnuplot ${ }^{59}$ to generate initial fitting parameters followed by a Markov Chain Monte Carlo (MCMC) method. The MCMC analysis is utilized to facilitate the computation of error bounds in the equilibrium length, i.e., the intersection between the fitting function for a particular knot type and for the phantom polygons, although the technique, inherently, also determines a mean fitting curve to the data. 
We use a slight variation on the Monte Carlo Markov chain (MCMC) technique described in ref 7. A detailed description of our method may be found in the Supporting Information for this work. For a given number of edges and fixed quantity, the distribution of the values is log-normal (which also is shown in the Supporting Information). The exceptions are the thickness radius (which appears to have an exponential distribution at each number of edges) and the total curvature and total torsion from ref 7 (which are normally distributed). The MCMC technique from ref 7 can be used with log-normal (and other distributions) but is computationally expensive. To speed the calculations we use the central limit theorem from statistics to transform the distributions that are not normal to a corresponding data set that is normally distributed.

For the sake of this study, it is easiest to explain the differences between the technique used here versus those reported in ref 7 by explaining one example. In measuring the miniball radius of the $3_{1}$ knots, the distribution of the data points is log-normal. For a given number of edges, we begin with a list of miniball radius values that are independent and unordered. We average the values (at each number of edges) in groups of 50 and discard any extra data points if the last group of numbers does not contain a full 50 data points. Next, we want to see if the averages are normally distributed so we use the Shapiro-Wilk test for normality at each number of edges. When fewer than $5 \%$ of the different numbers of edges fail the Shapiro-Wilk test, we conclude that the grouping has successfully transformed the log-normal distributions into normal distributions. If more than $5 \%$ fail the Shapiro-Wilk test, then we increase our grouping to 60 and return to our Shapiro-Wilk analysis. We continue to increase the groupings by 10 until we reach a grouping level where enough of the distributions are passing the Shapiro-Wilk test. In the end, we cut the data set size by a factor of 50 or more which in turn reduces the computation time by roughly the same factor. We then use the MCMC technique from ref 7 and the Supporting Information on the grouped data sets.

We ran some test cases using both the full set of data (using log-normal MCMC) and the grouped data (using Gaussian MCMC) and found that the error values obtained are similar (usually within 10\%) and that the latter error values always exceeded the former. In other words, our error values reported here are similar (likely slightly higher) than the error bounds we would obtain by running MCMC with the full log-normal data sets.

The MCMC calculations require a fitting function for the data. For most of the quantities, we used a function of the form $n^{d v}(A$ $+B / \sqrt{ } n+C / n)$, where $d=1,2,3$ is a measure of the dimension of the measurement and $v=0.5,0.588$ depends on whether we are measuring the attribute on phantom polygons or polygons of a fixed knot type. The two exceptions are the average crossing number and thickness radius.

For the average crossing number, we use the fitting function

$$
A\left(n-n_{K}\right) \ln \left(n-n_{K}\right)+B\left(n-n_{K}\right)+C
$$

for a given knot type (where $n_{K}$ is the minimum stick number for this knot type) and

$$
D n \ln (n)+E
$$

for the phantom polygons as in ref 6 .

For the thickness radius, the $\log -\log$ plot of the mean values is well fit by a function of the form $A+B / \sqrt{ } n+C / n$. Thus, we fit the thickness radius values with the function

$$
\exp (A+B / \sqrt{\ln (n)}+C / \ln (n))
$$

The MCMC calculations deliver 1000 likely fitting curves for each of the knot types (in addition to the phantom polygons) and spatial characteristics. From the fitting curves for a given quantity, we compute the 1000 intersections between the scaling profiles for the phantom polygons and those for each knot type. The largest and smallest $2.5 \%$ are discarded and the remaining values determine the $95 \%$ confidence intervals for the equilibrium length.

\section{Analysis}

In addition to the determination of the equilibrium lengths for various knot types, one of the objectives of this study is to determine whether imposing a given topology changes the scaling behavior of modeled polymeric chains with respect to quantities of very different character such as the overall dimensions, average crossing number, and thickness radius. In each case analyzed here, the scaling behavior of modeled polymers with unrestricted topology was different than that of modeled polymers confined to a given knot type. See Figure 1 for the case of miniball radius (MBR). The scaling profiles for individual knot types intersect the scaling profiles of phantom polymers to determine the equilibrium length of a given knot type with respect to the given characteristic. Table 1 lists the computed equilibrium length values that were calculated in this study as well as the values of the equilibrium length for the total curvature (TCU) and total torsion (TTO) from ref 7.

Our results show that there is significant visible structure among the values of the equilibrium lengths within the various categories of spatial characteristics. We see that there are four distinct families:

1. Average crossing number.

2. Radius of gyration and spatial dimensions.

3. Thickness radius.

4. Total curvature and total torsion.

The computed equilibrium lengths are shown in Table 1.

We have grouped the data to show that different quantities measuring a similar attribute of the knot population do, in fact, determine very similar equilibrium lengths. For example, we found that the total curvature and total torsion have equilibrium length values that are within, on average, $3.8 \%{ }^{7}$ Here, we find that the miniball radius, box length, and skinny box length, all measurements of the spatial extent of the knot, have their equilibrium length values within $0.4 \%$ on average. Furthermore, the equilibrium lengths with respect to the surface area and volume of both types of boxes and the convex hull are within $0.2 \%$. In fact, note that for the 14 quantities beginning with RGN in Table 1, the equilibrium lengths are within approximately $6 \%$, on average.

The equilibrium lengths form four distinct groups: (1) average crossing number, (2) radius of gyration and other spatial dimensions, (3) thickness radius, and (4) total curvature and total torsion. The differences between groups 3 and 4 are on the order of 7 to $8 \%$, on average. In the case of groups 1 and 2 , they differ by $28 \%$, on average. These differences suggest that the groups capture distinct spatial information. For the thickness radius, one might expect to find similarity with the miniball radius since a knot with small miniball radius must be tightly packed and, thus, have small thickness. We plot the equilibrium length derived from the thickness radius and average crossing number with respect to the equilibrium length derived from the miniball radius (Figure 3) and see that while the values are not the same, their relative values appear to be consistent.

For the average crossing number, we see equilibrium length values that are smaller than the majority of the quantities we computed. However, if we plot the equilibrium length with respect to the average crossing number versus the miniball radius, for example, we again see this nearly linear relationship in Figure 3. This nearly linear relationship exists between all the families of equilibrium values that we have computed. 


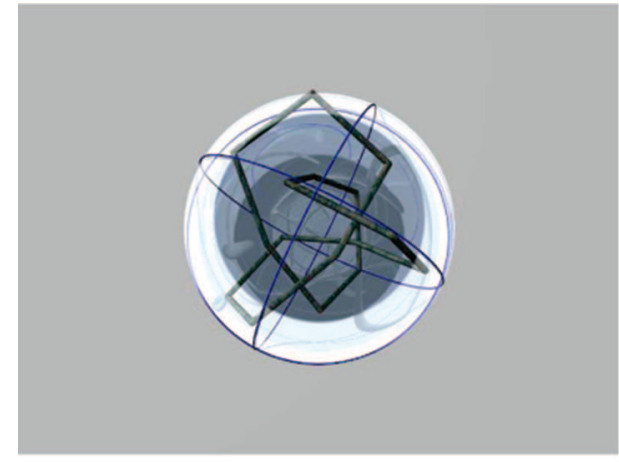

(a) Miniball

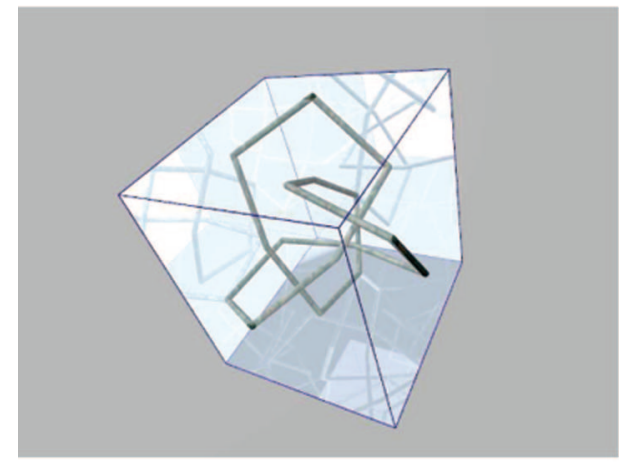

(c) Box

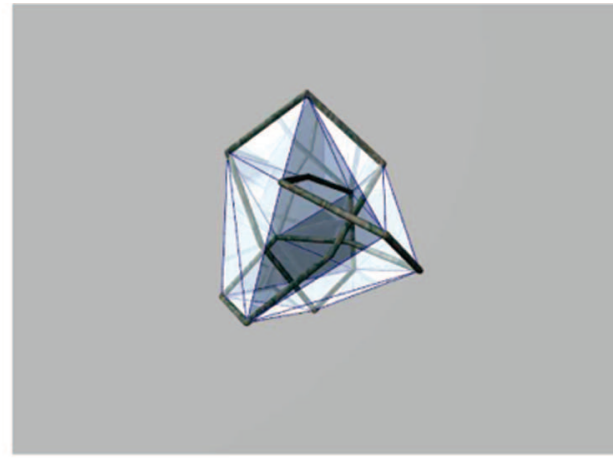

(b) Convex hull

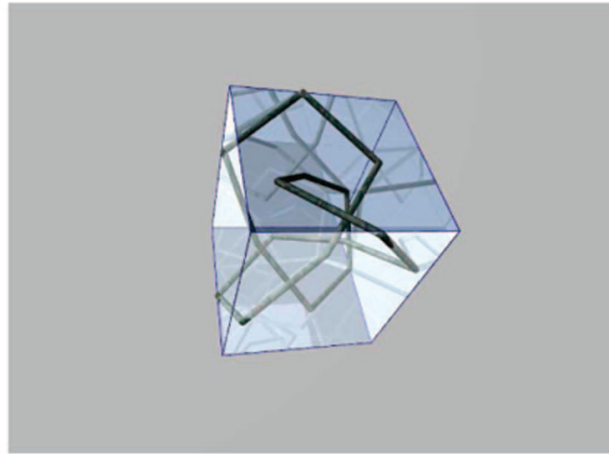

(d) Skinny box

Figure 2. Enclosing solids for a 16-edge figure-eight knot $\left(4_{1}\right)$ capture the knot's dimensions in different ways. The knot has been thickened for demonstration purposes, so the slight protrudance is to be expected.

Table 1. Equilibrium Lengths for the Different Knots with Respect to the Different Spatial Quantities

\begin{tabular}{|c|c|c|c|c|c|c|c|}
\hline & \multicolumn{7}{|c|}{ Equilibrium Length \pm Error } \\
\hline & $3_{1}$ & $4_{1}$ & $5_{1}$ & $5_{2}$ & $6_{1}$ & $6_{2}$ & 63 \\
\hline $\mathrm{ACN}$ & $169 \pm 1$ & $250 \pm 1$ & $337 \pm 2$ & $336 \pm 2$ & $431 \pm 5$ & $441 \pm 5$ & $447 \pm 7$ \\
\hline RGN & $187 \pm 1$ & $278 \pm 2$ & $368 \pm 5$ & $369 \pm 4$ & $475 \pm 12$ & $486 \pm 12$ & $487 \pm 16$ \\
\hline BXL & $191 \pm 1$ & $279 \pm 2$ & $363 \pm 5$ & $367 \pm 3$ & $461 \pm 10$ & $469 \pm 10$ & $474 \pm 12$ \\
\hline MBR & $191 \pm 1$ & $280 \pm 2$ & $363 \pm 4$ & $367 \pm 3$ & $460 \pm 9$ & $469 \pm 10$ & $472 \pm 13$ \\
\hline SBW & $195 \pm 1$ & $282 \pm 2$ & $366 \pm 5$ & $366 \pm 3$ & $460 \pm 11$ & $468 \pm 9$ & $474 \pm 13$ \\
\hline SBH & $198 \pm 1$ & $280 \pm 2$ & $359 \pm 5$ & $360 \pm 4$ & $436 \pm 11$ & $458 \pm 11$ & $451 \pm 14$ \\
\hline CHA & $198 \pm 1$ & $287 \pm 2$ & $370 \pm 3$ & $373 \pm 3$ & $465 \pm 7$ & $477 \pm 7$ & $478 \pm 9$ \\
\hline SBA & $198 \pm 1$ & $286 \pm 2$ & $371 \pm 3$ & $373 \pm 3$ & $464 \pm 7$ & $476 \pm 7$ & $477 \pm 10$ \\
\hline BXA & $198 \pm 1$ & $286 \pm 2$ & $370 \pm 3$ & $373 \pm 2$ & $465 \pm 8$ & $474 \pm 7$ & $480 \pm 12$ \\
\hline SBV & $203 \pm 1$ & $292 \pm 1$ & $377 \pm 4$ & $380 \pm 3$ & $469 \pm 7$ & $484 \pm 8$ & $484 \pm 11$ \\
\hline $\mathrm{CHV}$ & $203 \pm 1$ & $292 \pm 1$ & $376 \pm 3$ & $379 \pm 3$ & $471 \pm 7$ & $483 \pm 7$ & $483 \pm 10$ \\
\hline BXV & $203 \pm 1$ & $292 \pm 2$ & $377 \pm 4$ & $380 \pm 3$ & $471 \pm 8$ & $483 \pm 8$ & $490 \pm 12$ \\
\hline
\end{tabular}

This suggests a natural hypothesis: while the actual values of the equilibrium length may differ between spatial quantities, there exists a linear relationship between any pair. This would imply that the equilibrium length is a universal quantity, but scales depending on the particular quantity one measures.

We note an intriguing similarity between the probability distribution function (PDF) of a given characteristic for a given knot type near its equilibrium length with the corresponding PDF for phantom chains with the same length. Figure 5 shows that the PDF of the miniball radius (MBR) values for $3_{1}$ knots composed of 190 segments practically coincides with the PDF of MBR for phantom polygons with 190 segments. The means, of course, almost coincide as 190 segments is very close to the equilibrium length of trefoil knots with respect to MBR value (which is $191 \pm 1$, see Table 1). However, the fact that their distributions are so similar is surprising. The collection of phantom polygons contains approximately $22 \%$ trefoil knots, $49 \%$ unknots, and $29 \%$ more complex knots. The PDF of MBR for unknots with 190 segments is significantly shifted toward higher MBR values indicating that unknots are, on average, more expanded than trefoil knots or phantom polygons with the same chain size. In contrast, the PDF of MBR for knots more complex than the trefoil is shifted toward lower MBR values indicating that more complex knots are, on average, more compact than trefoil knots or phantom polygons. The fact that the PDFs for unknots and for the more complex knots are significantly shifted toward the right and left, respectively, compared to the trefoils may suggest that, by grouping all the knots together, the PDF of phantom polygons will be significantly broader than the PDF for the trefoil knots. However this is not what is observed. We 
observe only a slight difference in the variance $\left(0.93\right.$ for $3_{1}$ and 1.04 for phantom polygons). This behavior is a result of the balancing of the MBR values of the unknots by the more complex knots. For example, in the population of unknots, there is a higher fraction of more expanded configurations (large MBR values) than in the population of trefoil knots. On the other hand, in the population of more complex knots, there is a smaller fraction of expanded configurations than in the population of trefoils. As a consequence, when we add unknots and more complex knots to trefoil knots (obtaining the full population of phantom polygons), the fraction of expanded configurations stays the same as for the set of trefoil knots. The same principle, by analogy, applies to more compact configurations.

Within the range of our data, the profiles for the knot types never intersect. We believe, however, that some profiles may intersect for knots with higher crossing numbers, especially for knots that belong to different families (e.g., alternating and nonalternating, prime and composite, etc.). If this were not the case, then the ordering of the profiles would be entirely determined by the average values at every number of edges. However, at the minimal number of edges which is sufficient to create a certain knot type, the so-called stick number (see e.g., refs 60-65), the configurations can be quite constrained. At this number of edges, a particular knot type is likely to have anomalous average spatial values. As such, it would not be surprising to see intersections of the profiles for different knot types as the number of edges is increased and the knots are permitted to adopt more relaxed configurations. This will be more likely to happen for more complex knots as their corresponding profiles are expected to be much closer to each other than is seen for the seven knots types analyzed in this paper.

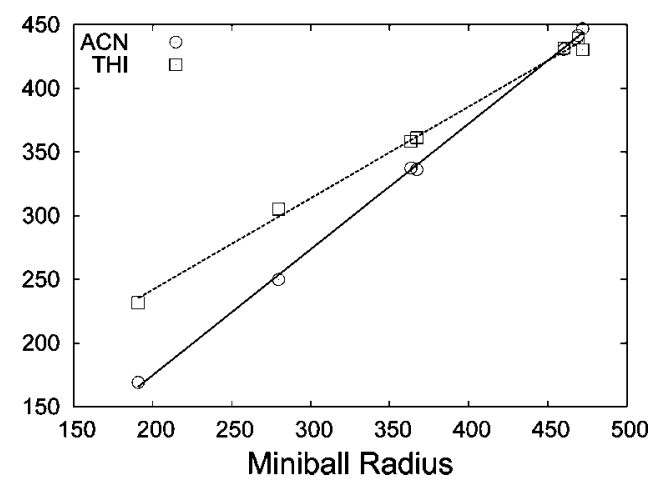

Figure 3. Equilibrium length based on thickness radius and average crossing number versus the equilibrium length based on miniball radius.

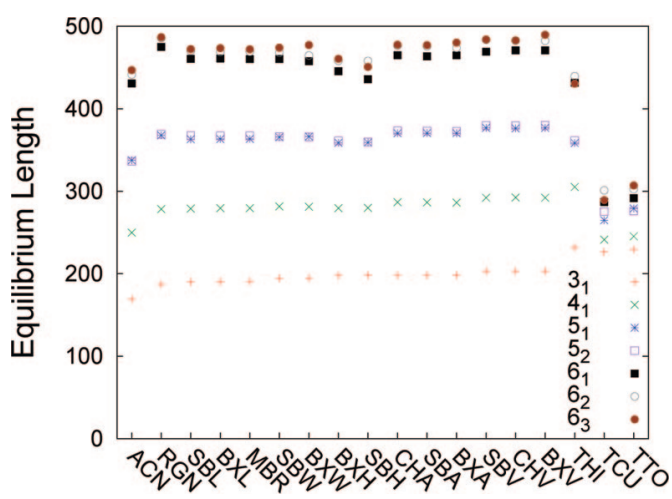

Figure 4. Equilibrium lengths for the different knots with respect to their distinct spatial quantities.

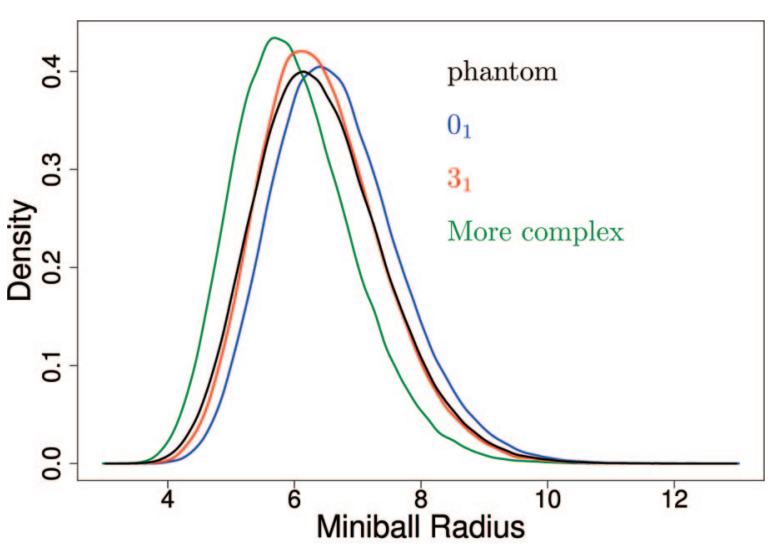

Figure 5. The density plots (i.e., estimations of the PDFs of the distributions) for the phantom polygons (black) and its constituent populations: $0_{1}$ knots (blue), $3_{1}$ knots (red), and the polygons which are more complex than the $3_{1}$ knot (green).

\section{Conclusions}

The results of our analysis are consistent with a scaling exponent, for the spatial characteristics studied here, of 0.5 for phantom polygons and 0.588 for knotted polygons, or an appropriate multiple. This is a consequence of the fact that random polymers with a fixed topology behave as if selfavoiding even though the excluded volume is set to zero. ${ }^{4,5,2,8}$

The equilibrium lengths of the radius of gyration and the new spatial characteristics investigated here form a distinct family suggesting that they reflect one spatial feature (Figure 4). Similarly, the equilibrium lengths associated to the total curvature and total torsion appear to form another distinct family thereby providing another spatial feature. The average crossing number and thickness radius equilibrium lengths appear to capture dimensions of the structure of polymers quite distinct from each other and from those captured by the first two families. Observing that the gaps between the average crossing number equilibrium length values for the different knot types appear to be similar to those for the new spatial characteristics (Figure 4), a linear relationship is suggested. This is confirmed, for these knots, as is seen in Figure 4 where the equilibrium length for the miniball radius is plotted versus the equilibrium lengths for the thickness radius and average crossing number.

The spatial characteristics we study here, with the exception of the average crossing number and thickness radius, would be expected to scale in a manner that depends upon the spatial nature of the characteristic due to the similarity of their equilibrium lengths to those of the radius of gyration. Thus, those that are linear spatial characteristics would scale in the same manner as the radius of gyration. The second order characteristics, those comparable to surface area, would have scaling exponents that are twice the linear exponent. Third order characteristics, comparable to volume, would have scaling exponents that are three times the linear exponents. One example, the box volume, is shown in Figure 6 where one can observe the scaling as well as the equilibrium length.

The two exceptions to this scaling behavior are the average crossing number $(\mathrm{ACN})$ and thickness radius (THI). While the average crossing number does not appear to have a scaling functional behavior, ${ }^{15}$ it has been shown to approach $A n \ln (n)$ (where $A$ is a fitting parameter) for very long polymers. For thickness radius, the $\log -\log$ plot was well fit by the function $A+B / \sqrt{ } n+C / n$ resulting in a fitting by $\exp (A+B / \sqrt{ } \ln (n)+$ $C / \ln (n))$.

Earlier studies revealed that average values of statistical characteristics of polymer chains forming various random knots are reflected in the shapes of the so-called ideal geometric 


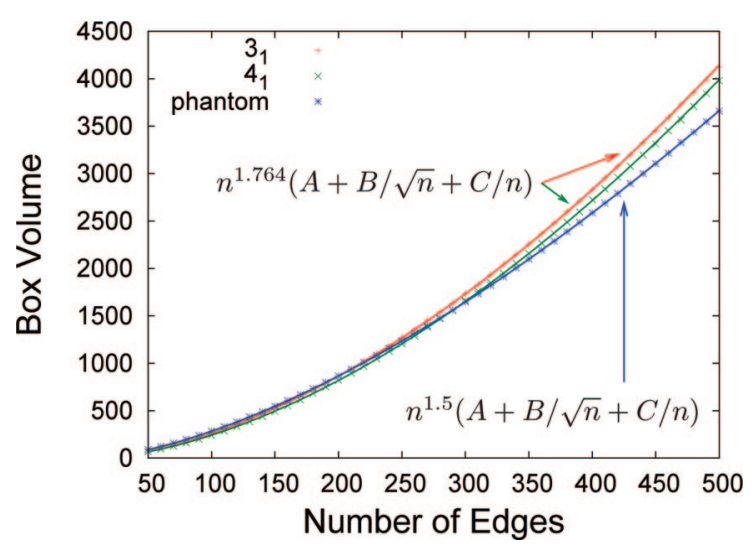

Figure 6. The scaling profiles for the box volume (BXV) for the $3_{1}$ and $4_{1}$ knots and the phantom polygons including the mean fitting function from the MCMC analysis. For the phantom polygons, $v$ is 0.5 , and for the knot types, $v$ is 0.588 .

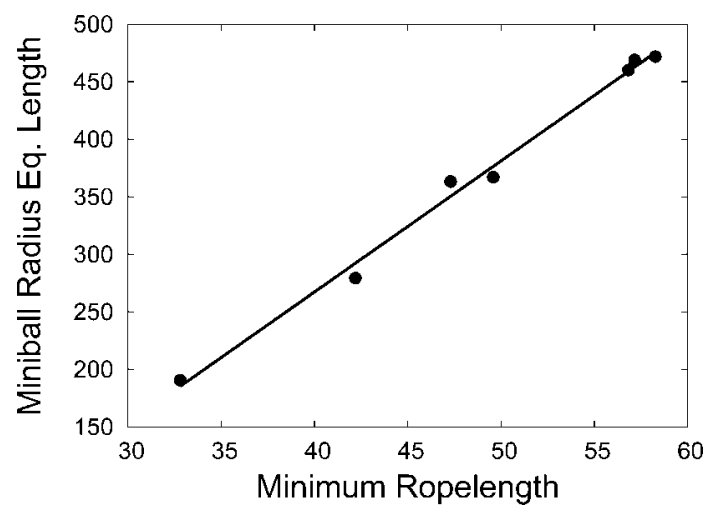

Figure 7. The miniball radius equilibrium lengths plotted versus the length of the ideal configurations from ref 74 .

configurations of knots. ${ }^{47,51,66,67}$ The ideal geometric configurations (see, e.g., refs 52 and 68-80) are the shapes needed for closing a given knot using a minimal length of flexible uniform radius rope and appear to be unique for simple knots. It was observed, for example, that the average writhe of a large statistical sample of random knots of a given knot type is very close to the writhe value of the computed ideal configuration. ${ }^{51,66}$ Also, a number of other statistical and physical properties of randomly fluctuating knots seem to be echoed by the ideal forms of these knots. For example, the electrophoretic migration speed of various knot types made of identical length DNA molecules was proportional to the average crossing number of the ideal configurations of the corresponding knot types. ${ }^{16,47}$ Depending on the radius of the rope, the overall dimensions of a given knot can vary, however its shape is invariant of scale as well as the ratio of the length and the radius of the rope needed to form a given knot. ${ }^{81}$

The ratio of length to radius is known as the ropelength of a knot and the minimum ropelength needed to form a given knot is a natural measurement of the complexity of a knot. The more complex the knot, the more rope is required to tie the knot. The equilibrium lengths studied here also show the same behavior since the more complex knots show higher equilibrium lengths and this is independent of the characteristic. This suggests that there could be a relationship between the equilibrium lengths and the ropelength of ideal configurations. Figure 7 shows that while the correlation is not perfect, one observes similar trends in both categories. Studies of equilibrium lengths of more complex knots would be needed to extend the plot and see if all points characterizing the relationship between an equilibrium length and the ropelength of ideal knots will follow the same power law function ${ }^{5}$ or whether different families of knots will behave differently and the corresponding points on the plot will fall within a narrow cone delimited by power law functions. $^{82}$

Acknowledgment. This research was supported in part by National Science Foundation Grant No. 0311010 for M.P., P.P., and E.J.R., by Swiss National Science Foundation Grants 3100A0116275 for A.S. and No. PA00A-105000 for A.D., and by the Institute for Mathematics and its Applications with funds provided by the National Science Foundation for K.C.M., E.J.R., and A.S. K.C.M. was also supported by the Centre de Mathematiques et d'Informatique, Marseille, France.

Supporting Information Available: Text describing the spatial quantities studied in this paper, detailed information about the MCMC computations, and figures showing histograms of the lognomial distributions for the quantities at a given number of edges. This material is available free of charge via the Internet at http:// pubs.acs.org.

\section{References and Notes}

(1) de Gennes, P. G. Scaling Concepts in Polymer Physics; Cornell University Press: Ithaca, NY, 1979.

(2) Grosberg, A. Y. Phys. Rev. Lett. 2000, 85, 3858

(3) Prellberg, T. J. Phys. A: Math. Gen. 2001, 34, L599.

(4) Deutsch, J. M. Phys. Rev. E 1999, 59, 2539.

(5) Dobay, A.; Dubochet, J.; Millett, K.; Sottas, P.-E.; Stasiak, A. Proc. Natl. Acad. Sci. U.S.A. 2003, 100, 5611.

(6) Diao, Y.; Dobay, A.; Kusner, R. B.; Millett, K. C.; Stasiak, A. J. Phys. A. 2003, 36, 11561 .

(7) Plunkett, P.; Piatek, M.; Dobay, A.; Kern, J.; Millett, K.; Stasiak, A.; Rawdon, E. Macromolecules 2007, 40, 3860.

(8) Moore, N. T.; Lua, R. C.; Grosberg, A. Y. Proc. Natl. Acad. Sci. U.S.A. 2004, 101, 13431.

(9) Shimamura, M. K.; Deguchi, T. J. Phys. A 2002, 35, 241.

(10) Frisch, H. L.; Wasserman, E. J. Am. Chem. Soc. 1961, 83, 3789.

(11) Delbrück, M. Mathematical Problems in the Biological Sciences; Proceedings of Symposia in Applied Mathematics 14; American Mathematical Society: Providence, RI, 1962; pp 55-63.

(12) Sumners, D. W.; Whittington, S. G. J. Phys. A 1988, 21, 1689.

(13) Pippenger, N. Discrete Appl. Math. 1989, 25, 273.

(14) Diao, Y. J. Knot Theory Ramifications 1995, 4, 189.

(15) Diao, Y.; Pippenger, N.; Sumners, D. W. J. Knot Theory Ramifications 1994, 3, 419

(16) Vologodskii, A. V.; Crisona, N. J.; Laurie, B.; Pieranski, P.; Katritch, V.; Dubochet, J.; Stasiak, A. J. Mol. Biol. 1998, $278,1$.

(17) Michels, J. P. J.; Wiegel, F. W. Proc. R. Soc. London Ser. A 1986, 403, 269.

(18) Orlandini, E.; Tesi, M. C.; Whittington, S. G.; Sumners, D. W.; Janse van Rensburg, E. J. J. Phys. A 1994, 27, L333.

(19) Orlandini, E.; Tesi, M. C.; Janse van Rensburg, E. J.; Whittington, S. G. J. Phys. A 1998, 31, 5953.

(20) Orlandini, E.; Tesi, M. C.; Whittington, S. G. J. Phys. A. 2005, 38, L795.

(21) Tsurusaki, K.; Deguchi, T. Statistical models, Yang-Baxter equation and related topics, and Symmetry, statistical mechanical models and applications (Tianjin, 1995); World Sci. Publ.: River Edge, NJ, 1996; pp 320-329.

(22) Deguchi, T.; Tsurusaki, K. Lectures at KNOTS' 96 (Tokyo); Knots Everything 15; World Sci. Publ.: River Edge, NJ, 1997; pp 95-122.

(23) Deguchi, T.; Tsurusaki, K. J. Knot Theory Ramifications 1994, 3, 321.

(24) Deguchi, T.; Tsurusaki, K. J. Phys. Soc. Jpn. 1993, 62, 1411.

(25) Janse van Rensburg, E. J.; Whittington, S. G. J. Phys. A 1990, 23, 3573.

(26) Janse van Rensburg, E. J.; Sumners, D. A. W.; Wasserman, E.; Whittington, S. G. J. Phys. A 1992, 25, 6557.

(27) Millett, K. C. J. Knot Theory Ramifications 1994, 3, 263.

(28) Calvo, J. A.; Millett, K. C. Ideal knots; Knots Everything 19; World Sci. Publ.: River Edge, NJ, 1998; pp 107-128.

(29) Millett, K. C. Knots in Hellas '98 (Delphi); Knots Everything 24; World Sci. Publ.: River Edge, NJ, 2000; pp 306-334.

(30) Moore, N. T.; Lua, R. C.; Grosberg, A. Y. In Physical and Numerical Models in Knot Theory; Calvo, J. A., Millett, K. C., Rawdon, E. J., Stasiak., A., Eds.; Knots Everything 36; World Sci. Publishing: Singapore, 2005; pp 363-384. 
(31) Moore, N. T.; Lua, R. C.; Grosberg, A. Y. In Physical and Numerical Models in Knot Theory; Calvo, J. A., Millett, K. C., Rawdon, E. J., Stasiak, A., Eds.; Knots Everything 36; World Sci. Publishing: Singapore, 2005; pp 385-398.

(32) Adams, C. C. The knot book; American Mathematical Society: Providence, RI, 2004; An elementary introduction to the mathematical theory of knots, Revised reprint of the 1994 original.

(33) Rolfsen, D. Knots and links; Mathematics Lecture Series 7; Publish or Perish Inc.: Houston, TX, 1990. Corrected reprint of the 1976 original.

(34) Miniball, http://miniball.sourceforge.net/. Program for computing smallest enclosing ball.

(35) Flammini, A.; Maritan, A.; Stasiak, A. Biophys. J. 2004, 87, 2968.

(36) Stasiak, A. Encyclopedia of the Human Genome; Nature Publishing Group: London, 2003.

(37) Dean, F.; Stasiak, A.; Koller, T.; Cozzarelli, N. J. Biol. Chem. 1985 $260,4975$.

(38) Rybenkov, V. V.; Ullsperger, C.; Vologodskii, A. V.; Cozzarelli, N. R. Science 1997, 277, 690

(39) Buck, G. R.; Lynn Zechiedrich, E. J. Mol. Biol. 2004, 340, 933.

(40) Liu, Z.; Mann, J. K.; Zechiedrich, E. L.; Chan, H. S. J. Mol. Biol. 2006, 361, 268

(41) Banchoff, T. Indiana Univ. Math. J. 1976, 25, 1171.

(42) Brakke, K.; Sullivan, J. Surface Evolver, http://www.geom.uiuc.edu/ software/download/. Program for visualizing and energy minimizing knots.

(43) Cimasoni, D J. Knot Theory Ramifications 2001, 10, 387.

(44) Rawdon, E. J. The Thickness of Polygonal Knots. Ph.D. thesis, University of Iowa, Iowa City, IA, 1997.

(45) Rawdon, E. J. Ideal knots; World Sci. Publishing: Singapore 1998; pp 143-150.

(46) Rawdon, E. J. J. Knot Theory Ramifications 2000, 9, 113

(47) Stasiak, A.; Katritch, V.; Bednar, J.; Michoud, D.; Dubochet, J. Nature 1996, 384, 122

(48) Stasiak, A.; Dubochet, J.; Katritch, V.; Pieranski, P. Ideal knots; World Sci. Publishing: Singapore, 1998; pp 1-19.

(49) Maritan, A.; Micheletti, C.; Trovato, A.; Banavar, J. R. Nature 2000, 406, 287.

(50) Grosberg, A. Y. Ideal knots; Knots Everything 19; World Sci Publishing: Singapore, 1998; pp 129-142.

(51) Cerf, C.; Stasiak, A. Proc. Natl. Acad. Sci. U.S.A. 2000, 97, 3795.

(52) Millett, K. C.; Rawdon, E. J. J. Comput. Phys. 2003, 186, 426.

(53) qhull: http://www.qhull.org. Program for computing the convex hull.

(54) Fenchel, W. Ann. Math. 1929, 101, 238.

(55) Milnor, J. W. Ann. Math. 1950, 52, 248.

(56) Klenin, K. V.; Vologodskii, A. V.; Anshelevich, V. V.; Dykhne, A. M.; Frank-Kamenetskii, M. D. J. Biomol. Struct. Dyn. 1988, 5, 1173.
(57) Freyd, P.; Yetter, D.; Hoste, J.; Lickorish, W. B. R.; Millett, K.; Ocneanu, A. Bull. Amer. Math. Soc. (N.S.) 1985, 12, 239.

(58) Ewing, B.; Millett, K. C. Progress in knot theory and related topics; Hermann: Paris, 1997; pp 51-68.

(59) gnuplot: http://www.gnuplot.info. Program for plotting and fitting data.

(60) Randell, R. J. Knot Theory Ramifications 1993, 3, 279.

(61) Meissen, M. Knot theory; Polish Acad. Sci.: Warsaw, 1995; pp $235-242$

(62) Adams, C. C.; Brennan, B. M.; Greilsheimer, D. L.; Woo, A. K. J. Knot Theory Ramifications 1997, 6, 149.

(63) McCabe, C. L. J. Knot Theory Ramifications 1998, 7, 797.

(64) Rawdon, E. J.; Scharein, R. G. Physical knots: knotting, linking, and folding geometric objects in R 3; Contemp. Math. 304; Amer. Math. Soc.: Providence, RI, 2002; pp 55-75.

(65) Furstenberg, E.; Li, J.; Schneider, J. Chaos Solitons Fractals 1998, 9 , 561. Knot theory and its applications.

(66) Katritch, V.; Bednar, J.; Michoud, D.; Scharein, R. G.; Dubochet, J.; Stasiak, A. Nature 1996, 384, 142.

(67) Pieranski, P.; Przbyl, S. Eur. Phys. J. E 2001, 6, 117.

(68) Diao, Y.; Ernst, C.; Janse van Rensburg, E. J. J. Knot Theory Ramifications 1997, 6, 799.

(69) Diao, Y.; Ernst, C.; Janse van Rensburg, E. J. Math. Proc. Cambridge Philos. Soc. 1999, 126, 293.

(70) Pierański, P. Ideal knots; World Sci. Publishing: Singapore, 1998; pp $20-41$.

(71) Diao, Y. J. Knot Theory Ramifications 2003, 12, 1.

(72) Pieranski, P.; Przybyl, S. Phys. Rev. E 2001, 64, 031801-5.

(73) Litherland, R. A.; Simon, J.; Durumeric, O.; Rawdon, E. Topology Appl. 1999, 91, 233.

(74) Rawdon, E. J. Experiment. Math. 2003, 12, 287.

(75) Cantarella, J.; Kusner, R. B.; Sullivan, J. M. Invent. Math. 2002, 150, 257.

(76) Baranska, J.; Pieranski, P.; Przybyl, S.; Rawdon, E. J. Phys. Rev. E 2004, 70, 051810.

(77) Gonzalez, O.; Maddocks, J. H. Proc. Natl. Acad. Sci. USA 1999, 96, 4769.

(78) Ashton, T.; Cantarella, J.; Piatek, M.; Rawdon, E. Self-contact sets for 50 tightly knotted and linked tubes; arXiv:math.DG/0508248.

(79) Baranska, J.; Pieranski, P.; Rawdon, E. J. In Physical and Numerical Models in Knot Theory; Calvo, J. A., Millett, K. C., Rawdon, E. J., Stasiak, A., Eds.; Knots Everything 36; World Sci. Publishing: Singapore, 2005; pp 293-321.

(80) Denne, E.; Diao, Y.; Sullivan, J. M. Geom. Topol. 2006, 10, 1.

(81) Grosberg, A. Y.; Feigel, A.; Rabin, Y. Phys. Rev. E 1996, 54, 6618.

(82) Buck, G. Nature 1998, 392, 238. Scientific Correspondence.

MA8000803 